BIR-QMW/PH/91-1.

\title{
Hidden Kac-Moody symmetry and 2D Quantum Supergravity
}

\author{
W.A. Sabra \\ Physics Department \\ Birkbeck College \\ University of London \\ Malet Street \\ London WC1E $7 H X$ \\ and \\ Physics Department \\ Queen Mary and Westfield College \\ University of London \\ Mile End Road \\ London E1 $4 N S$ \\ United Kingdom
}

\begin{abstract}
We examine the relation between Polyakov's formulation of two dimensional supergravity and gauged Wess-Zumino-Novikov-Witten models.
\end{abstract}




\section{Introduction}

The subject of two dimensional (super)conformal field theory has connections with many active areas of both theoretical physics and pure mathematics. In particular, two dimensional (super)conformal field theory coupled to two dimensional (super)gravity is not only of interest as a tractable theory of quantum (super)gravity but an essential ingredient in the formulation of off-critical (super)string theories in the description of elementary particles. From the point of view of statistical mechanics, two dimensional quantum gravity describes the critical theory at a second order phase transition point on a random lattice.

The subject was transformed by Polyakov's treatment of two dimensional gravity as an induced theory arising from quantum averaging over some underlying matter degrees of freedom coupling to a gravitational field via their stress-energy tensor [1].

It is clear from the work of Polyakov et al. [1], that one can gain a more understanding of the problem of quantizing two dimensional quantum gravity if, instead of the conformal gauge, one chooses alternatively a "chiral" gauge. For this choice, there are analogies one can make between two dimensional quantum gravity and the WessZumino-Novikov-Witten (WZNW) model [16]. This then led Polyakov to the remarkable discovery that the theory possesses an associated chiral current algebra based upon the non-compact group $S L(2, R)$. This result was obtained by an explicit calculation of the correlation functions for the gravitational field. A generalization of this result to both the $N=1$ and $N=2$ supersymmetric case is possible [1,7], yielding graded versions of $S L(2, R)$, the orthogonal symplectic groups $O S p(1,2)$ and $O S p(2,2)$ respectively.

Recently, there has been a lot of progress in understanding the symmetries of Polyakov's two dimensional gravity and supergravities in terms of the coadjoint orbits method (for a review see [11]) and the gauged Wess-Zumino-Novikov-Witten models. Alekseev and Shatashvili [2] constructed a geometric action on a particular coadjoint orbit of the Virasoro group [3]. The action they derived is,

$$
S_{v i r}(F)=\frac{k}{2 \pi} \int d \lambda 2 z \frac{\partial_{\bar{z}} F}{\left(\partial_{z} F\right) \lambda 3}\left(\partial_{z} F \partial_{z} \lambda 3 F-2\left(\partial_{z} \lambda 2 F\right) \lambda 2\right),
$$

where $\partial_{z}=\partial / \partial z, \partial_{\bar{z}}=\partial / \partial_{\bar{z}}$. ${ }^{*}$ The action $S_{v i r}(F)$ is invariant under the $S L(2, R)$ transformation symmetry,

$$
F(z, \bar{z}) \rightarrow \frac{d(\bar{z}) F+b(\bar{z})}{c(\bar{z}) F+a(\bar{z})} ; \quad a d-b c=1 .
$$

In addition, in reference [2] it was shown that the action (1.1) is classically equivalent to a Borel gauged WZNW model with the gauge group $S L(2, R)$. The equivalence at the quantum level was demonstrated by Bershadsky and Ooguri [4] by showing that the representations of the conformal field theory are those of the constrained $S L(2, R)$ WZNW model.

The two dimensional quantum gravity action can be obtained from the geometric action (1.1) through the following transformation,

$$
F(f(z, \bar{z}), \bar{z})=z
$$

* we parametrize the two dimensional space time with coordinates $(x, t)$ by $z=t+x$ and $\bar{z}=t-x$. 
and is expressed in terms of the scalar field $f$ as,

$$
S_{\text {grav }}\left(h_{\bar{z} \bar{z}}\right)=-\frac{k}{2 \pi} \int d \lambda 2 z\left(\frac{\partial_{z} \lambda 2 f \partial_{z} \partial_{\bar{z}} f}{\left(\partial_{z} f\right) \lambda 2}-\frac{\left(\partial_{z} \lambda 2 f\right) \lambda 2 \partial_{\bar{z}} f}{\left(\partial_{z} f\right) \lambda 3}\right), \quad h_{\bar{z} \bar{z}}=\frac{\partial_{\bar{z}} f}{\partial_{z} f}
$$

where $h_{\bar{z} \bar{z}}$ is the component of the gravitational field surviving the light-cone gauge.

With regard to the supersymmetric extensions of these results, an $(1,0)$ supersymmetric geometric action has been formulated in $[5,6]$ on the coadjoint orbit (of purely central extension) of the $N=1$ superVirasoro group. The authors also derive an action for $(1,0)$ supergravity by gauging the Borel subgroup of the $(0,1)$ supersymmetric $S L(2, R)$ WZNW model. However, the supergravity action they obtained does not have the $O S p(1,2)$ current algebra but instead an $N=1$ supersymmetric extension of the $S L(2, R)$ current algebra. This formulation of $(1,0)$ supergravity [5,6], simply corresponds to a different gauge choice in the $(1,0)$ supergravity theory, and is explained as follows. In $(1,0)$ supergravity the covariant derivatives are expressed as,

$$
\nabla_{A}=E_{A} \lambda M D_{M}+\omega_{A} M
$$

where $E_{A} \lambda M$ are the vielbiens and $\omega_{A}$ is the connection. The torsion and curvature are determined by the graded commutators of the covariant derivatives. In order to reduce the component fields of supergravity, one must introduce a set of algebraic constraints on these superfields. For $(1,0)$ supergravity, the unconstrained fields [23] are, in component form,

$$
\begin{aligned}
H \lambda \bar{z}_{\theta} & =\rho_{\theta} \lambda \bar{z}+\theta h \lambda \bar{z}_{z}, \\
H \lambda z_{\bar{z}} & =h \lambda z_{\bar{z}}+\theta \psi \lambda \theta_{\bar{z}}, \\
S & =h+\theta \psi \lambda \theta_{\bar{z}} .
\end{aligned}
$$

The component fields $\left\{h \lambda z_{\bar{z}}, h \lambda \bar{z}_{z}, h\right\}$ represent the graviton, $\left\{\psi \lambda \theta_{\bar{z}}, \psi \lambda \theta_{z}\right\}$ represent the gravitino and $\rho \lambda \bar{z}_{\theta}$ is a pure gauge degree of freedom. Choosing the chiral gauge, $H \lambda \bar{z}_{\theta}=0, S=1$, gives an action whose degrees of freedom are the graviton and the gravitino, and the equation of motion derived from the anomaly equation $[7,25]$ is,

$$
\partial \lambda 2_{z} D_{\theta} H \lambda z_{\bar{z}}=0
$$

The symmetry of the theory in the above chiral gauge was derived in [7] where it was shown that the system possesses an $O S p(1,2)$ current algebra. However, if we choose instead a different gauge, by setting $H \lambda z_{\bar{z}}=0, S=1$, the action obtained describes the graviton and the gauge degree of freedom $\rho \lambda \bar{z}_{\theta}$. In this gauge, the equation of motion derived from the anomaly equation is,

$$
\partial \lambda 3_{\bar{z}} H \lambda \bar{z}_{\theta}=0
$$

This equation is nothing more than the supersymmetrization of the bosonic gravity equation of motion $\partial_{\bar{z}} \lambda 3 h \lambda \bar{z}_{z}$. It is easily realized that one obtains an $N=1, S L(2, R)$ current algebra, where the $S L(2, R)$ currents of the bosonic case [1] are promoted to superfields with fermionic components related to the extra degree of freedom $\rho \lambda \bar{z}_{\theta}$. This corresponds to the model considered in $[5,6]$. 
The $(1,0)$ geometric action, constructed on the coadjoint orbit of purely central extension of the superVirasoro group is given by $[5,6,11]$,

$$
S_{s . v i r} \lambda(1,0)=-\frac{k}{4 \pi} \int d \lambda 2 z d \theta\left(\frac{\partial_{\bar{z}} Z_{0}+\Theta_{0} \partial_{\bar{z}} \Theta_{0}}{\left(D_{\theta} \Theta_{0}\right) \lambda 2}\right)\left(\frac{D_{\theta} \lambda 4 \Theta_{0}}{D_{\theta} \Theta_{0}}-3 \frac{D_{\theta} \lambda 3 \Theta_{0}}{D_{\theta} \Theta_{0}} \frac{D_{\theta} \lambda 2 \Theta_{0}}{D_{\theta} \Theta_{0}}\right)
$$

where $Z_{0}(z, \bar{z}, \theta)$ and $\Theta_{0}(z, \bar{z}, \theta)$ are superdiffeomorphisms in $(z, \theta)$ superspace obeying the superconformal condition,

$$
D_{\theta} Z_{0}=\Theta_{0} D_{\theta} \Theta_{0}
$$

where $D_{\theta}=\partial_{\theta}+\theta \partial_{z}$ is the superderivative. By using the superconformal condition, the geometric action (1.9) can now be rewritten in the form,

$$
S_{\text {s.vir }} \lambda(1,0)=\frac{k}{2 \pi} \int d \lambda 2 z d \theta \frac{\partial_{\bar{z}} \Theta_{0} D_{\theta} \lambda 3 \Theta_{0}}{\left(D_{\theta} \Theta_{0}\right) \lambda 2} .
$$

The $(1,1)$ geometric action can be deduced simply by replacing $\partial_{\bar{z}}$ with the superderivative $D_{\bar{\theta}}=\partial_{\bar{\theta}}+\bar{\theta} \partial_{\bar{z}}$ in Eq. (1.11),

$$
S_{\text {s.vir }} \lambda(1,1)=\frac{k}{2 \pi} \int d \lambda 2 z d \lambda 2 \theta \frac{D_{\bar{\theta}} \Theta D_{\theta} \lambda 3 \Theta}{\left(D_{\theta} \Theta\right) \lambda 2} .
$$

The relation of the above action to that of $(1,1)$ supergravity [12] is obtained through the following set of transformations,

$$
X(\phi, \bar{z}, \psi, \bar{\theta})=z, \quad \Theta(\phi, \bar{z}, \psi, \bar{\theta})=\theta
$$

The $(1,1)$ supergravity action is then given as $[12,25]$,

$$
S\left(H \lambda z_{\bar{\theta}}\right)=-\frac{k}{2 \pi} \int d \lambda 2 z d \lambda 2 \theta \frac{\partial_{z} \psi}{\left(D_{\theta} \psi\right) \lambda 2}\left(D_{\bar{\theta}}-\frac{D_{\bar{\theta}} \phi-\psi D_{\bar{\theta}} \psi}{\left(D_{\theta} \psi\right) \lambda 2} \partial_{z}\right) D_{\theta} \psi,
$$

where the gravitational field is parametrized as,

$$
H \lambda z_{\bar{\theta}}=\frac{D_{\bar{\theta}} \phi-\psi D_{\bar{\theta}} \psi}{\left(D_{\theta} \psi\right) \lambda 2}
$$

It has been argued in [9], that the geometric actions (1.11) and (1.12) are equivalent to those obtained by gauging the Borel subgroup of $(1,0)$ and $(1,1)$ super $S L(2, R)$ WessZumino-Novikov-Witten models respectively. However, in the analysis of [9], it is not quite clear how the superconformal condition can be obtained from the gauged WZNW model.

In [10], the geometric action (1.11) was shown to be equivalent to the Borel gauged $\operatorname{OSp}(1,2)$ WZNW model. There it was found that the constraints obtained after integrating out the gauge field are equivalent to the superconformal condition.

Following the analysis of [10], we shall show that the action (1.12) describes a theory equivalent to a Borel gauged $(0,1) O S p(1,2)$ WZNW model. We will also explain the origin of the relation (Eq. (1.13)) which connects the geometric action (1.12) and $(1,1)$ supergravity action and derive the supergravitational "composition formula" [13]. The 
relation between the central charge of the $O S p(1,2)$ current algebra of $(1,1)$ induced supergravity and the conformal anomaly of the superconformal matter is also obtained.

This work is organised as follows. In section 2 , the formulation of $(1,1)$ suprgravity in the superlight cone gauge $[1,7,8]$ is briefly reviewed. In section 3 we show the equivalence of the Borel gauged $(0,1) O S p(1,2)$ WZNW model to that of $S \lambda(1,1)_{\text {s.vir }}$. Section 4 is concerned in showing the relation of the geometric action to that of $(1,1)$ supergravity. In the last section a canonical derivation of the renormalization of the central charge of the current algebra of supergravity is given.

\section{Review of $(1,1)$ Supergravity}

In this section the theory of $(1,1)$ supergravity formulated in the super light-cone gauge is briefly reviewed. The $(1,1)$ supergravity theory is described by a set of covariant derivatives $[7,20]$

$$
\nabla_{A}=E_{A} \lambda M D_{M}+\omega_{A} M
$$

where $E_{A} \lambda M$ are the vielbeins, $w_{A}$ are the spin connections and $M$ is the Lorentz generator. The superspace derivative $D_{A}$ is,

$$
D_{A}=\left(\partial_{z}, \partial_{\bar{z}}, D_{\theta}=\frac{\partial}{\partial \theta}+\theta \partial_{z}, D_{\bar{\theta}}=\frac{\partial}{\partial \bar{\theta}}+\bar{\theta} \partial_{\bar{z}}\right)
$$

The constraints in the $(1,1)$ supergravity theory are,

$$
\begin{aligned}
& \left\{\nabla_{\theta}, \nabla_{\theta}\right\}=2 \nabla_{z}, \\
& \left\{\nabla_{\bar{\theta}}, \nabla_{\bar{\theta}}\right\}=2 \nabla_{\bar{z}}, \\
& \left\{\nabla_{\theta}, \nabla_{\bar{\theta}}\right\}=R M .
\end{aligned}
$$

As a consequence of the constraints, some of the components $E_{A} \lambda M$ and $\omega_{A}$ are expressed in terms of a set of independent superfields. After solving the constraints and going to the super light-cone gauge, it is found that the only degree of freedom in the theory is $H_{\bar{\theta}} \lambda z$ and the covariant derivatives are expressed in terms of $H \lambda z_{\bar{\theta}}$ as,

$$
\begin{aligned}
\nabla_{\bar{\theta}} & =D_{\bar{\theta}}-H \lambda z_{\bar{\theta}} \partial_{z}+\frac{1}{2}\left(D_{\theta} H \lambda z_{\bar{\theta}}\right) D_{\theta}-\partial_{z} H \lambda z_{\bar{\theta}} M, \\
\nabla_{\theta} & =D_{\theta} .
\end{aligned}
$$

The equation of motion for the field $H \lambda z_{\bar{\theta}}$, derived from the anomaly equation is,

$$
D_{\theta} \partial_{z} \lambda 2 H \lambda z_{\bar{\theta}}=0 \text {. }
$$

The solution to the equation of motion (2.4) can be expressed in terms of superfield currents as,

$$
\begin{aligned}
H \lambda z_{\bar{\theta}}(z, \bar{z}, \theta, \bar{\theta})= & \mathcal{J} \lambda+1(\bar{z}, \bar{\theta})-2 \mathcal{J} \lambda 0(\bar{z}, \bar{\theta}) z+\mathcal{J} \lambda-1(\bar{z}, \bar{\theta}) z \lambda 2 \\
& +\theta\left(\mathcal{J} \lambda \frac{1}{2}(\bar{z}, \bar{\theta})-\mathcal{J} \lambda-\frac{1}{2}(\bar{z}, \bar{\theta}) z\right)
\end{aligned}
$$


From the calculation of the Ward identity involving the gravitational field $H \lambda z_{\bar{\theta}}$, it can be deduced that these currents satisfy an $N=1$ super $\operatorname{OSp}(1,2)$ Kac-Moody algebra [21],

$$
\mathcal{J} \lambda a\left(\bar{z}_{1}, \bar{\theta}_{1}\right) \mathcal{J} \lambda b\left(\bar{z}_{2}, \bar{\theta}_{2}\right)=-\frac{\hat{k} / 2 \eta \lambda a b}{\bar{Z}_{12}}+\frac{\bar{\theta}_{12} f \lambda a b_{c} \mathcal{J} \lambda c\left(\bar{z}_{2}, \bar{\theta}_{2}\right)}{\bar{Z}_{12}},
$$

where $\hat{k}$ is the central charge of the current algebra, $\bar{Z}_{12}=\bar{z}_{1}-\bar{z}_{2}-\bar{\theta}_{1} \bar{\theta}_{2}$ and $\bar{\theta}_{12}=\bar{\theta}_{1}-\bar{\theta}_{2}$, $\eta \lambda a b$ is the $O S p(1,2)$ invariant metric and $f \lambda a b_{c}$ are the structure constants of $O S p(1,2)$ algebra. After fixing the super light-cone gauge, the theory is invariant only under residual transformations respecting the gauge choice. It can be shown that these residual transformations are generated by $T \lambda$ total, $\mathcal{J} \lambda-1$ and $\mathcal{J} \lambda-\frac{1}{2}$, where,

$$
T \lambda t o t a l=T \lambda s . m a t t+T \lambda s . g r a v+T \lambda g h o s t
$$

is the sum of the contributions of the superconformal matter, the gravitational field and the ghost fields to the stress energy tensor. It has to be noted that $T \lambda s . g r a v$ has, besides the Sugawara form [24], an additional linear term in the current $\mathcal{J} \lambda 0$,

$$
T \lambda s . g r a v=T \lambda s u g+\partial_{\bar{z}} \mathcal{J} \lambda 0 .
$$

This modified stress tensor satisfies a superVirasoro algebra with conformal anomaly

$$
c \lambda s . g r a v=\left(\frac{1}{2}+\frac{2 k}{2 k+3}\right)-6\left(k+\frac{3}{2}\right) ; \quad k=-\hat{k}-\frac{3}{2}
$$

where the first term comes from the Sugawara form [24] and the second term derives from the linear term.

The central charge of the current algebra is determined by the coefficient which multiplies the induced quantum supergravity action and supergravitational quantum fluctuations may lead to a finite renormalization of this central charge. To calculate this requires summing up all loop contributions to the effective action. Instead, however, one can use $[1,8]$ the self consistency relation arising from the residual invariance of the theory. This relation is satisfied if the total central charge of the theory vanishes, i.e,

$$
\begin{aligned}
c \lambda \text { total } & =c \lambda s . m a t t+c \lambda s . g r a v+c \lambda \text { ghost } \\
& =c \lambda s . m a t t+\frac{1}{2}+\frac{2 k}{2 k+3}-6\left(k+\frac{3}{2}\right)-9 \\
& =0
\end{aligned}
$$

where -9 is the sum of the conformal anomalies of the ghosts introduced to fix the super light-cone gauge and $c \lambda s$.matt $=3 d / 2$ is the conformal anomaly of the superconformal matter inducing quantum supergravity.

Details of the study of $(1,1)$ supergravity in the super light-cone gauge are given in $[8]$. 


\section{Borel Gauged $(0,1) \operatorname{OSp}(1,2)$ WZNW model}

We start by reviewing the Borel gauged $O S p(1,2)$ WZNW model [10]. The resulting model is equivalent to the geometric action related to $(1,0)$ induced supergravity.

Let $\operatorname{OSp}(1,2)$ denote the set of graded matrices $M$ satisfying,

$$
M \lambda t \sigma_{1}+(-1) \lambda \kappa \sigma_{1} M=0
$$

where $\kappa=0$ for the even elements of $M$ and $\kappa=1$ for the odd elements, $\sigma_{1}$ is given by,

$$
\sigma_{1}=\left(\begin{array}{ccc}
0 & -1 & 0 \\
1 & 0 & 0 \\
0 & 0 & 1
\end{array}\right)
$$

and $M \lambda t$ denotes the supertranspose of the graded matrix $M$. Eq. (3.1) is solved by,

$$
M=\left(\begin{array}{ccc}
m & n & -\zeta \\
p & -m & \chi \\
\chi & \zeta & 0
\end{array}\right)
$$

where $\{m, n, p\}$ are even and $\{\zeta, \chi\}$ are odd elements.

The following basis of $\operatorname{OSp}(1,2)$ is now considered,

$$
\begin{aligned}
l_{0} & =\left(\begin{array}{ccc}
\frac{1}{2} & 0 & 0 \\
0 & -\frac{1}{2} & 0 \\
0 & 0 & 0
\end{array}\right) l_{1}=\left(\begin{array}{ccc}
0 & 1 & 0 \\
0 & 0 & 0 \\
0 & 0 & 0
\end{array}\right) l_{-1}=\left(\begin{array}{lll}
0 & 0 & 0 \\
1 & 0 & 0 \\
0 & 0 & 0
\end{array}\right) \\
l_{-\frac{1}{2}} & =\left(\begin{array}{lll}
0 & 0 & 0 \\
0 & 0 & 1 \\
1 & 0 & 0
\end{array}\right) l_{\frac{1}{2}}=\left(\begin{array}{ccc}
0 & 0 & 1 \\
0 & 0 & 0 \\
0 & -1 & 0
\end{array}\right) .
\end{aligned}
$$

The Borel subgroup is defined as the subgroup generated by $l_{-1}$ and $l_{-\frac{1}{2}}$. The Borel gauged $O S p(1,2)$ WZNW model is described by the following action,

$$
S_{\text {gauged }}=S(g)+\operatorname{str} \int d \lambda 2 z V_{1}\left(\partial_{z} g g \lambda-1-K_{1}\right),
$$

where $g(z, \bar{z}) \in O S p(1,2)$, str denotes the supertrace and $S(g)$ is the original $O S p(1,2)$ WZNW model [16]. The supermatrix $V_{1}$ is a gauge field taking values in the Borel algebra of $O S p(1,2)$ and plays the role of a Lagrange multiplier and $K_{1}$ is a constant supermatrix,

$$
V_{1}=\left(\begin{array}{ccc}
0 & 0 & 0 \\
A & 0 & \eta \\
\eta & 0 & 0
\end{array}\right) \quad K_{1}=\left(\begin{array}{ccc}
0 & 1 & 0 \\
0 & 0 & 0 \\
0 & 0 & 0
\end{array}\right) .
$$

The $O S p(1,2)$ group element $g$ can be represented by,

$$
g=\left(\begin{array}{ccc}
1 & 0 & 0 \\
F_{1} & 1 & e_{1} \\
e_{1} & 0 & 1
\end{array}\right)\left(\begin{array}{ccc}
F_{2} & 0 & 0 \\
0 & F_{2} \lambda-1 & 0 \\
0 & 0 & 1
\end{array}\right)\left(\begin{array}{ccc}
1 & F_{3} & e_{2} \\
0 & 1 & 0 \\
0 & -e_{2} & 1
\end{array}\right)
$$


where $\left\{F_{1}, F_{2}, F_{3}\right\}$ are bosonic fields while $\left\{e_{1}, e_{2}\right\}$ are fermionic fields.

In expressing the gauged action in terms of the components fields it is very useful to use the Polyakov-Wiegmann identity [15],

$$
S\left(g_{1} g_{2}\right)=S\left(g_{1}\right)+S\left(g_{2}\right)+\frac{k}{2 \pi} \int d \lambda 2 z \operatorname{str}\left(g_{1} \lambda-1 \partial_{\bar{z}} g_{1} \partial_{z} g_{2} g_{2} \lambda-1\right)
$$

Integrating over the gauge field in (3.5) imposes the constraints,

$$
\begin{gathered}
F_{2} \lambda 2\left(\partial_{z} F_{3}+\partial_{z} e_{2} e_{2}\right)=1, \\
F_{2} \partial_{z} e_{2}-e_{1}=0
\end{gathered}
$$

which, after being substituted back into the gauged action and using the Borel local gauge invariance to gauge away the field $\Phi$, leaves a reduced theory which can be expressed only in terms of the dynamical fields $F_{3}$ and $e_{2}$ in the following reduced form,

$$
S\left(F_{3}, e_{2}\right)=\frac{k}{2 \pi} \int d \lambda 2 z\left(F_{2} \lambda-1 \partial_{z} F_{2} F_{2} \lambda-1 \partial_{\bar{z}} F_{2}-F_{2} \lambda 2 \partial_{z} e_{2} \partial_{\bar{z}} \partial_{z} e_{2}\right)
$$

supplemented by (3.9a).

We now turn to the action (1.11) and express it in component form. In terms of the components of $\Theta_{0}$,

$$
\Theta_{0}=\alpha_{0}(z, \bar{z})+\theta \beta_{0}(z, \bar{z})
$$

the action (1.11) can be expressed as,

$$
S_{s . v i r} \lambda(1,0)=\frac{k}{2 \pi} \int d \lambda 2 z\left(\frac{\partial_{\bar{z}} \beta_{0} \partial_{z} \beta_{0}}{\beta_{0} \lambda 2}+\frac{\partial_{z} \partial_{\bar{z}} \alpha_{0} \partial_{z} \alpha_{0}}{\beta_{0} \lambda 2}\right) .
$$

If we now make the identification

$$
\beta_{0}=\frac{1}{F_{2}}, \quad \alpha_{0}=i e_{2},
$$

then the action (3.10) coincides with (3.12).

The action (1.11) is supplemented with the superconformal condition (1.10) which, in terms of the components of $\Theta_{0}$ and $Z_{0}=X_{0}+\theta X_{1}$, gives,

$$
\partial_{z} X_{0}=-\alpha_{0} \partial_{z} \alpha_{0}+\beta_{0} \lambda 2 .
$$

Making the identification $X_{0}=F_{3}$, (3.14) becomes the constraint (3.9a) which supplements the reduced action (3.10).

Now it is straightforward to generalize the above analysis to the Borel gauged $(0,1)$ $O S p(1,2)$ WZNW model. Here the model is described by three $(0,1)$ bosonic superfields $\left\{\mathcal{F}_{1}, \mathcal{F}_{2}, \mathcal{F}_{3}\right\}$ and two fermionic $(0,1)$ superfields $\left\{\mathcal{E}_{1}, \mathcal{E}_{2}\right\}$. action

The Borel gauged $(0,1) O S p(1,2)$ WZNW model is described by the following

$$
S_{\text {gauged }}=S(G)+\operatorname{str} \int d \lambda 2 z d \bar{\theta} V\left(\partial_{z} G G \lambda-1-K_{1}\right)
$$


where $G(z, \bar{z}, \bar{\theta})$ is a group element of $O S p(1,2), S(G)$ is the original $(0,1) O S p(1,2)$ WZNW model and the supermatrix $V_{1}$ is a gauge superfield taking values in the Borel algebra of $O S p(1,2)$ and plays the role of a Lagrange multiplier.

In expressing the gauged action in terms of the components fields, it is very useful to employ the supersymmetric version of the Polyakov-Wiegmann identity,

$$
S\left(G_{1} G_{2}\right)=S\left(G_{1}\right)+S\left(G_{2}\right)+\frac{k}{2 \pi} \int d \lambda 2 z d \bar{\theta} \operatorname{str}\left(G_{1} \lambda-1 D_{\bar{\theta}} G_{1} \partial_{z} G_{2} G_{2} \lambda-1\right)
$$

Following the same steps as for the bosonic case, we express the gauged action in terms of the dynamical superfields $\mathcal{F}_{3}$ and $\mathcal{E}_{2}$ in the following reduced form,

$$
S\left(\mathcal{F}_{3}, \mathcal{E}_{2}\right)=\frac{k}{2 \pi} \int d \lambda 2 z d \bar{\theta}\left(\mathcal{F}_{2} \lambda-1 \partial_{z} \mathcal{F}_{2} \mathcal{F}_{2} \lambda-1 D_{\bar{\theta}} \mathcal{F}_{2}-\mathcal{F}_{2} \lambda 2 \partial_{z} \mathcal{E}_{2} \partial_{z} D_{\bar{\theta}} \mathcal{E}_{2}\right)
$$

supplemented by,

$$
\mathcal{F}_{2} \lambda 2\left(\partial_{z} \mathcal{F}_{3}+\partial_{z} \mathcal{E}_{2} \mathcal{E}_{2}\right)=1
$$

The gauged action is invariant under the right symmetry $G \rightarrow G Q^{\prime}(\bar{z}, \bar{\theta})$. We represent $Q^{\prime}(\bar{z}, \bar{\theta}) \in O S p(1,2)$ by,

$$
Q^{\prime}(\bar{z}, \bar{\theta})=\left(\begin{array}{ccc}
a & b & \alpha \\
c & d & \beta \\
a \beta-c \alpha & b \beta-d \alpha & 1+\beta \alpha
\end{array}\right)
$$

where $\{a, b, c, d\}$ are even and $\{\alpha, \beta\}$ are odd elements, with $(a d-b c=1-\beta \alpha)$. Therefore, the reduced action is invariant under,

$$
\begin{aligned}
\mathcal{F}_{2} & \rightarrow \frac{d \mathcal{F}_{2}+b}{c \mathcal{F}_{2}+a}+\frac{\left(\beta \mathcal{F}_{2}+\alpha\right) \mathcal{E}_{2}}{\left(c \mathcal{F}_{2}+a\right) \lambda 2}, \\
\mathcal{E}_{2} & \rightarrow \frac{\alpha+\beta \mathcal{F}_{2}}{c \mathcal{F}_{2}+a}+\frac{\mathcal{E}_{2}}{c \mathcal{F}_{2}+a}
\end{aligned}
$$

These are the super $O S p(1,2)$ transformations. The maximal symmetry in the left moving part of the theory is the superconformal symmetry [19].

In conclusion, the Borel gauged $(0,1) O S p(1,2)$ model has the same symmetries as the $S \lambda(1,1)_{\text {s.vir }}$ model, i.e., a super $O S p(1,2)$ Kac-Moody symmetry in the rightmoving sector and superdiffeomorphism in the the left moving part of the theory.

We now turn to the action (1.12) and express it in terms of the component fields. Expanding $\Theta=\Theta_{0}(z, \bar{z}, \theta)+\bar{\theta} \Theta_{1}(z, \bar{z}, \theta)$, the action (1.12) can be expressed in the form,

$$
S_{s . v i r} \lambda(1,1)=\frac{k}{2 \pi} \int d \lambda 2 z d \theta\left(\frac{\partial_{\bar{z}} \Theta_{0} \partial_{z} D_{\theta} \Theta_{0}}{\left(D_{\theta} \Theta_{0}\right) \lambda 2}+\frac{\partial_{z} \Theta_{1} D_{\theta} \Theta_{1}}{\left(D_{\theta} \Theta_{0}\right) \lambda 2}\right)
$$

In terms of the components of $\Theta_{0}$ and $\Theta_{1}$,

$$
\begin{aligned}
& \Theta_{0}=\alpha_{0}(z, \bar{z})+\theta \beta_{0}(z, \bar{z}), \\
& \Theta_{1}=\alpha_{1}(z, \bar{z})+\theta \beta_{1}(z, \bar{z}),
\end{aligned}
$$


the action (3.21) becomes,

$S_{s . v i r} \lambda(1,1)=\frac{k}{2 \pi} \int d \lambda 2 z\left(\frac{\partial_{\bar{z}} \beta_{0} \partial_{z} \beta_{0}}{\beta_{0} \lambda 2}+\frac{\partial_{z} \partial_{\bar{z}} \alpha_{0} \partial_{z} \alpha_{0}}{\beta_{0} \lambda 2}-2 \frac{\partial_{z} \alpha_{0} \partial_{z} \alpha_{1} \beta_{1}}{\beta_{0} \lambda 3}+\left(\frac{\partial_{z} \alpha_{1}}{\beta_{0}}\right) \lambda 2+\frac{\partial_{z} \beta_{1} \beta_{1}}{\beta_{0} \lambda 2}\right)$

We also express the reduced action (3.17) in components. Writing $\mathcal{F}_{2}=\lambda_{1}+\bar{\theta} \lambda_{2}$ and $\mathcal{E}_{2}=f_{1}+\bar{\theta} f_{2}$, the reduced action (3.17) is expressed,

$$
\begin{array}{r}
S\left(\mathcal{F}_{3}, \mathcal{E}_{2}\right)=\frac{k}{2 \pi} \int d \lambda 2 z\left(\frac{\partial_{z} \lambda_{1} \partial_{\bar{z}} \lambda_{1}+\partial_{z} \lambda_{2} \lambda_{2}}{\lambda_{1} \lambda 2}-2 \lambda_{1} \lambda_{2} \partial_{z} f_{1} \partial_{z} f_{2}\right. \\
\left.+\lambda_{1} \lambda 2 \partial_{z} f_{1} \partial_{\bar{z}} \partial_{z} f_{1}-\lambda_{1} \lambda 2\left(\partial_{z} f_{2}\right) \lambda 2\right)
\end{array}
$$

Now making the identification,

$$
\beta_{0}=\frac{1}{\lambda_{1}}, \quad \alpha_{0}=i f_{1}, \quad \alpha_{1}=i f_{2}, \quad \beta_{1}=\frac{\lambda_{2}}{\lambda_{1} \lambda 2}
$$

the action (3.24) is found to coincide with (3.23).

The action (1.12) is supplemented with the superconformal condition,

$$
D_{\theta} Z=\Theta D_{\theta} \Theta ; \quad Z=Z_{0}+\bar{\theta} Z_{1}
$$

which gives,

$$
\begin{gathered}
D_{\theta} Z_{0}=\Theta_{0} D_{\theta} \Theta_{0}, \\
D_{\theta} Z_{1}=-\Theta_{0} D_{\theta} \Theta_{1}-\Theta_{1} D_{\theta} \Theta_{0} .
\end{gathered}
$$

Expanding $Z_{0}$ and $Z_{1}$ as follows,

$$
Z_{0}=X_{0}+\theta X_{1}, \quad Z_{1}=Y_{0}+\theta Y_{1}
$$

Eq. (3.26) yields,

$$
\begin{gathered}
\partial X_{0}=-\alpha_{0} \partial \alpha_{0}+\beta_{0} \lambda 2 \\
\partial Y_{0}=-2 \beta_{0} \beta_{1}-\alpha_{1} \partial \alpha_{0}+\alpha_{0} \partial \alpha_{1} .
\end{gathered}
$$

The constraint (3.18) supplementing the reduced action (3.17), in components, gives,

$$
\begin{gathered}
\lambda_{1} \lambda 2\left(\partial_{z} F_{1}+\partial_{z} f_{1} f_{1}\right)-1=0, \\
\lambda_{1} \lambda 2\left(\partial_{z} F_{2}+\partial_{z} f_{2} f_{1}-\partial_{z} f_{1} f_{2}\right)+2 \frac{\lambda_{2}}{\lambda_{1}}=0,
\end{gathered}
$$

where $\mathcal{F}_{3}=F_{1}+\bar{\theta} F_{2}$. Identifying $F_{1}$ and $F_{2}$ with $X_{0}$ and $Y_{0}$ respectively and using (3.25), equations (3.27a) and (3.27b) are shown to coincide with (3.28a) and (3.28b) respectively. The above demonstration shows the equivalence of the two theories.

\section{Gauge transformations and superdiffeomorphisms}

In the introduction it was stated that the $(1,1)$ supergravity action (Eq. (1.14)) is connected to the action $S_{\text {s.vir }} \lambda(1,1)$ through a change of variables (Eq. (1.13)). In 
this section, this connection is explained by exploiting the relation between superdiffeomorphisms and restricted gauge transformations [13]. We first derive the $(1,1)$ supersymmetric version of the Polyakov-Wiegmann identity, the so called "composition formula" [15]. Then from the relation between superdiffeomorphisms and restricted gauge transformations [13], the connection between the geometric action $S_{\text {s.vir }} \lambda(1,1)$ and $S_{\text {s.grav }} \lambda(1,1)$ is verified, appearing to arise from a consequence of a simple property of WZNW models. Finally the "composition formula" for the $(1,1)$ supergravity action is obtained, which is also reminiscent of that for the WZNW model.

Consider a $(1,1)$ supersymmetric two dimensional gauge theory with an $O S p(1,2)$ gauge group. This theory is described in terms of two gauge fields $A_{\theta} \lambda a$ and $A_{\bar{\theta}} \lambda a$, where $a=\left(1,0,-1, \frac{1}{2},-\frac{1}{2}\right)$ is an $\operatorname{SSp}(1,2)$ gauge group index. The gauge transformations of the gauge fields are given as,

$$
\begin{aligned}
& \delta A_{\theta} \lambda a=-\mathcal{D}_{\theta} \epsilon \lambda a=-\left(D_{\theta} \epsilon \lambda a-f \lambda a_{b c} A_{\theta} \lambda b \epsilon \lambda c\right), \\
& \delta A_{\bar{\theta}} \lambda a=-\mathcal{D}_{\bar{\theta}} \epsilon \lambda a=-\left(D_{\bar{\theta}} \epsilon \lambda a-f \lambda a_{b c} A_{\bar{\theta}} \lambda b \epsilon \lambda c\right),
\end{aligned}
$$

where $f \lambda a_{b c}$ are the structure constants of the $O S p(1,2)$ algebra.

The action of the gauge theory described by $A_{\theta}$, is given by,

$$
S_{1}\left(A_{\theta}\right) \sim \log \operatorname{sdet}\left(D_{\theta}-A_{\theta}\right) .
$$

Its variation under gauge transformations is now,

$$
\delta S_{1}\left(A_{\theta}\right)=\int d \lambda 2 z d \lambda 2 \theta \operatorname{str}\left(J_{\bar{\theta}} \delta A_{\theta}\right)
$$

where $J_{\bar{\theta}}$ is the gauge current satisfying the anomaly equation

$$
\mathcal{D}_{\theta} J_{\bar{\theta}}=-k D_{\bar{\theta}} A_{\theta} .
$$

By substituting (4.1a) in (4.3) and using the above anomaly equation, we obtain

$$
\begin{aligned}
\delta S_{1}\left(A_{\theta}\right) & =-\int d \lambda 2 z d \lambda 2 \theta \operatorname{str}\left(J_{\bar{\theta}} \mathcal{D}_{\theta} \epsilon\right) \\
& =-\int d \lambda 2 z d \lambda 2 \theta \operatorname{str}\left(\epsilon \mathcal{D}_{\theta} J_{\bar{\theta}}\right) \\
& =k \int d \lambda 2 z d \lambda 2 \theta \operatorname{str}\left(\epsilon D_{\bar{\theta}} A_{\theta}\right) .
\end{aligned}
$$

We now Parametrize $A_{\theta}$ by,

$$
A_{\theta}=D_{\theta} g g \lambda-1
$$

where $g$ is a group element of $O S p(1,2)$ gauge group in $(1,1)$ superspace. Then $S_{1}\left(A_{\theta}\right)$ is solved by a $(1,1)$ super WZNW model, $S_{1}(g)$.

Similarly one can parametrize $A_{\bar{\theta}}=D_{\bar{\theta}} h h \lambda-1$, where $h$ is a group element of $O S p(1,2)$ gauge group in $(1,1)$ superspace, and study the dynamics of the gauge field $A_{\bar{\theta}}$, to find that,

$$
S_{2}\left(A_{\bar{\theta}}\right) \sim \log \operatorname{sdet}\left(D_{\bar{\theta}}-A_{\bar{\theta}}\right)
$$


is also solved by an $N=1 O S p(1,2)$ WZNW model, $S_{2}(h)$. The final form of the total effective action is then,

$$
S_{e f f}(g, h)=S_{1}(g)+S_{2}(h)-k \int d \lambda 2 z d \lambda 2 \theta \operatorname{str}\left(D_{\theta} g g \lambda-1 D_{\bar{\theta}} h h \lambda-1\right)
$$

where the last term is added to insure gauge invariance. Now, a finite gauge transformation on $A_{\theta}$ and $A_{\bar{\theta}}$ is given by,

$$
g \rightarrow U g, \quad h \rightarrow U h, \quad U \in O S p(1,2)
$$

As the effective action (4.8) is invariant under this transformation, this implies the following relation,

$$
S_{e f f}(g, h)=S_{e f f}(U g, U h) .
$$

If we set $U=h \lambda-1$ or $U=g \lambda-1$, we can then deduce that

$$
S_{e f f}(g, h)=S_{1}(h \lambda-1 g)=S_{2}(g \lambda-1 h),
$$

and in particular,

$$
S_{1}(h \lambda-1)=S_{2}(h)
$$

Finally, we arrive at the $(1,1)$ supersymmetric extension of the Polyakov-Weigmann composition formula,

$$
S_{1}(h \lambda-1 g)=S_{1}(g)+S_{1}(h \lambda-1)-k \int d \lambda 2 z d \lambda 2 \theta \operatorname{str}\left(D_{\theta} g g \lambda-1 D_{\bar{\theta}} h h \lambda-1\right) .
$$

Following Polyakov [13], we now partially fix a gauge by imposing the following conditions,

$$
A_{\theta} \lambda 1=A_{\theta} \lambda 0=A_{\theta} \lambda-\frac{1}{2}=0, \quad A_{\theta} \lambda \frac{1}{2}=1 .
$$

Then the gauge transformations (4.1), give the following relations,

$$
\begin{gathered}
\delta A_{\theta} \lambda-1=-D_{\theta} \epsilon \lambda-1+A_{\theta} \lambda-1 \epsilon \lambda 0, \\
\delta A_{\theta} \lambda 1=-D_{\theta} \epsilon \lambda 1-2 \epsilon \lambda \frac{1}{2}=0, \\
\delta A_{\theta} \lambda 0=-D_{\theta} \epsilon \lambda 0-2 A_{\theta} \lambda-1 \epsilon \lambda 1+2 \epsilon \lambda-\frac{1}{2}=0, \\
\delta A_{\theta} \lambda \frac{1}{2}=-D_{\theta} \epsilon \lambda \frac{1}{2}-\frac{1}{2} \epsilon \lambda 0=0, \\
\delta A_{\theta} \lambda-\frac{1}{2}=-D_{\theta} \epsilon \lambda-\frac{1}{2}+A_{\theta} \lambda-1 \epsilon \lambda \frac{1}{2}-\epsilon \lambda-1=0,
\end{gathered}
$$

which then give the following relations among the gauge parameters,

$$
\epsilon \lambda 0=-2 D_{\theta} \epsilon \lambda \frac{1}{2}=\partial_{z} \epsilon \lambda 1
$$




$$
\epsilon \lambda-1=\frac{1}{2} A_{\theta} \lambda-1 D_{\theta} \epsilon \lambda 1-D_{\theta} A_{\theta} \lambda-1 \epsilon \lambda 1-\frac{1}{2} \partial_{z} \epsilon \lambda 0 .
$$

These relations, when substituted back into (4.15a) now give,

$$
\delta A_{\theta} \lambda-1=+\frac{1}{2} D_{\theta} \partial \lambda 2_{z} \epsilon \lambda 1+\frac{1}{2} D_{\theta} A \lambda-1_{\theta} D_{\theta} \epsilon \lambda 1+\partial_{z} A_{\theta} \lambda-1 \epsilon \lambda 1+\frac{3}{2} A_{\theta} \lambda-1 \partial_{z} \epsilon \lambda 1 .
$$

If we set $T_{\theta z}=k A_{\theta} \lambda-1$, we then obtain the following equation

$$
\delta T_{\theta z}=\frac{k}{2} D_{\theta} \partial \lambda 2_{z} \epsilon \lambda 1+\frac{1}{2} D_{\theta} T_{\theta z} D_{\theta} \epsilon \lambda 1+\partial_{z} T_{\theta z} \epsilon \lambda 1+\frac{3}{2} T_{\theta z} \partial \epsilon \lambda 1
$$

This relation represents the infinitesimal superconformal transformation of the superstress energy tensor. This is because within the background of $A_{\theta} \lambda \frac{1}{2}=1$, the isospin is equivalent to the spin and thus all the fields in this background will acquire an additional spin equals to their $O S p(1,2)$ isospin [13]. Therefore $A \lambda-1_{\theta}$ is a spin $3 / 2$ superfield and also, $\epsilon \lambda 1$ can be identified with $\epsilon \lambda z$. In the partially gauge fixed theory discussed above, the WZNW model will become a model describing the dynamics of $T_{\theta z}$ and so $\delta S\left(A_{\theta}\right)$ becomes,

$$
\delta S\left(T_{\theta z}\right)=\int d \lambda 2 z d \lambda 2 \theta \epsilon \lambda z D_{\bar{\theta}} T_{\theta z}
$$

where $\delta S\left(T_{\theta z}\right)$ represents the transformation under the superconformal transformation (4.18). In addition, $\delta S\left(T_{\theta z}\right)$ is given by,

$$
\delta S\left(T_{\theta z}\right)=-\int d \lambda 2 z d \lambda 2 \theta L \lambda z_{\bar{\theta}} \delta T_{\theta z}
$$

where $L \lambda z_{\bar{\theta}}$ is a function of $T_{z \theta}$. Comparing the above two equations and using (4.18), we deduce that $L \lambda z_{\bar{\theta}}$ should satisfy the following equation,

$$
\left(D_{\bar{\theta}}-L \lambda z_{\bar{\theta}} \partial_{z}+\frac{1}{2}\left(D_{\theta} L \lambda z_{\bar{\theta}}\right) D_{\theta}-\frac{3}{2}\left(\partial_{z} L \lambda z_{\bar{\theta}}\right)\right) T_{\theta z}=-\frac{k}{2} D_{\theta} \partial \lambda 2_{z} L \lambda z_{\bar{\theta}}
$$

Define $S\left(H \lambda z_{\bar{\theta}}\right)$ as the Legendre transform of $S\left(T_{\theta z}\right)$ [13], then its transformation under superdiffeomorphisms is given by,

$$
\delta S\left(H \lambda z_{\bar{\theta}}\right)=\int d \lambda 2 z d \lambda 2 \theta Z_{\theta z} \delta H \lambda z_{\bar{\theta}}
$$

where $Z_{\theta z}$ satisfies the following equation,

$$
\left(D_{\bar{\theta}}-H \lambda z_{\bar{\theta}} \partial_{z}+\frac{1}{2}\left(D_{\theta} H \lambda z_{\bar{\theta}}\right) D_{\theta}-\frac{3}{2}\left(\partial_{z} H \lambda z_{\bar{\theta}}\right)\right) Z_{\theta z}=-\frac{k}{2} D_{\theta} \partial \lambda 2_{z} H \lambda z_{\bar{\theta}}
$$

and the field $H \lambda z_{\bar{\theta}}$ satisfies the following transformation,

$$
\delta H \lambda z_{\bar{\theta}}=\left(D_{\bar{\theta}}-H \lambda z_{\bar{\theta}} \partial_{z}+\frac{1}{2}\left(D_{\theta} H \lambda z_{\bar{\theta}}\right) D_{\theta}-\left(\partial_{z} H \lambda z_{\bar{\theta}}\right)\right) \epsilon \lambda z
$$


Finally, we define the action

$$
W\left(H \lambda z_{\bar{\theta}}, T_{\theta z}\right)=S\left(H \lambda z_{\bar{\theta}}\right)+S\left(T_{\theta z}\right)-\int d \lambda 2 z d \lambda 2 \theta H \lambda z_{\bar{\theta}} T_{\theta z} .
$$

It can be easily checked that this combined action is invariant under the transformations given by equations (4.18) and (4.24).

We turn now to find a solution for the action $S\left(H \lambda z_{\bar{\theta}}\right)$. Parametrize $H \lambda z_{\bar{\theta}}$ as,

$$
H \lambda z_{\bar{\theta}}=\frac{D_{\bar{\theta}} \phi-\psi D_{\bar{\theta}} \psi}{\left(D_{\theta} \psi\right) \lambda 2}
$$

where $\phi$ and $\psi$ satisfy,

$$
D_{\theta} \phi=\psi D_{\theta} \psi,
$$

then the anomaly equation (4.21) is solved by a superSchwartzian derivative,

$$
Z_{\theta z}=k\left(\frac{\partial \lambda 2_{z} \psi}{D_{\theta} \psi}-2 \frac{\partial_{z} \psi \partial_{z} D_{\theta} \psi}{\left(D_{\theta} \psi\right) \lambda 2}\right)=k \mathcal{S}(\psi)
$$

and the action $S\left(H \lambda z_{\bar{\theta}}\right)$ is given by,

$S\left(H \lambda z_{\bar{\theta}}\right)=-k S_{s . g r a v} \lambda(1,1)(\phi, \psi)=-k \int d \lambda 2 z d \lambda 2 \theta \frac{\partial_{z} \psi}{\left(D_{\theta} \psi\right) \lambda 2}\left(D_{\bar{\theta}}-\frac{D_{\bar{\theta}} \phi-\psi D_{\bar{\theta}} \psi}{\left(D_{\theta} \psi\right) \lambda 2} \partial_{z}\right) D_{\theta} \psi$

The action $S\left(T_{\theta z}\right)$ is the geometric quantization of the superVirasoro algebra, and it is given by,

$$
\begin{aligned}
S\left(T_{\theta z}\right) & =k S_{s . v i r} \lambda(1,1)=k \int d \lambda 2 z d \lambda 2 \theta\left(\frac{D_{\bar{\theta}} \Theta D_{\theta} \lambda 3 \Theta}{\left(D_{\theta} \Theta\right) \lambda 2}\right) \\
& =-\frac{k}{2} \int d \lambda 2 z d \lambda 2 \theta\left(\frac{D_{\bar{\theta}} X-\Theta D_{\bar{\theta}} \Theta}{\left(D_{\theta} \Theta\right) \lambda 2}\right)\left(\frac{\partial \lambda 2_{z} \Theta}{D_{\theta} \Theta}-3 \frac{\partial_{z} \Theta \partial_{z} D_{\theta} \Theta}{\left(D_{\theta} \Theta\right) \lambda 2}\right),
\end{aligned}
$$

where $X(z, \bar{z}, \theta, \bar{\theta})$ and $\Theta(z, \bar{z}, \theta, \bar{\theta})$ satisfy the superconformal condition

$$
D_{\theta} X=\Theta D_{\theta} \Theta,
$$

and

$$
\begin{aligned}
L \lambda z_{\theta} & =\frac{D_{\bar{\theta}} X-\Theta D_{\bar{\theta}} \Theta}{\left(D_{\theta} \Theta\right) \lambda 2} \\
T_{\theta z} & =k\left(\frac{\partial \lambda 2_{z} \Theta}{D_{\theta} \Theta}-2 \frac{\partial_{z} \Theta \partial_{z} D_{\theta} \Theta}{\left(D_{\theta} \Theta\right) \lambda 2}\right)=k \mathcal{S}(\Theta) .
\end{aligned}
$$

The finite form of (4.18) and (4.24) can be represented by

$$
\begin{gathered}
X(z, \bar{z}, \theta, \bar{\theta}) \rightarrow X\left(X_{1}, \bar{z}, \Theta_{1}, \bar{\theta}\right), \\
\Theta(z, \bar{z}, \theta, \bar{\theta}) \rightarrow \Theta\left(X_{1}, \bar{z}, \Theta_{1}, \bar{\theta}\right), \\
\phi(z, \bar{z}, \theta, \bar{\theta}) \rightarrow \phi\left(X_{1}, \bar{z}, \Theta_{1}, \bar{\theta}\right), \\
\psi(z, \bar{z}, \theta, \bar{\theta}) \rightarrow \psi\left(X_{1}, \bar{z}, \Theta_{1}, \bar{\theta}\right),
\end{gathered}
$$


which symbolically will be written as

$$
(X, \Theta) \rightarrow(X, \Theta) \bullet\left(X_{1}, \Theta_{1}\right), \quad(\phi, \psi) \rightarrow(\phi, \psi) \bullet\left(X_{1}, \Theta_{1}\right)
$$

These relations are obviously the gravitational analogue of,

$$
g \rightarrow U g, \quad h \rightarrow U h
$$

Also $(X \lambda-1, \Theta \lambda-1)$ is defined as,

$$
(X, \Theta) \bullet(X \lambda-1, \Theta \lambda-1)=(z, \theta) .
$$

This to be understood as the gravitational analogue of $g g \lambda-1=I$, where $I$ is the identity.

The invariance of the combined action $W\left(H \lambda z_{\bar{\theta}}, T_{\theta z}\right)$ under superdiffeomorphisms implies the relationship,

$$
W((X, \Theta),(\phi, \psi))=W\left((X, \Theta) \bullet\left(X_{1}, \Theta_{1}\right),(\phi, \psi) \bullet\left(X_{1}, \Theta_{1}\right)\right)
$$

If we set $\left(X_{1}, \Theta_{1}\right)=(\phi \lambda-1, \psi \lambda-1)$ or $\left(X_{1}, \Theta_{1}\right)=(X \lambda-1, \Theta \lambda-1)$, then the above equation gives

$$
\begin{aligned}
W((X, \Theta),(\phi, \psi)) & =k S_{s . v i r} \lambda(1,1)((X, \Theta) \bullet(\phi \lambda-1, \psi \lambda-1)) \\
& =-k S_{\text {s.grav }} \lambda(1,1)((\phi, \psi) \bullet(X \lambda-1, \Theta \lambda-1))
\end{aligned}
$$

and in particular,

$$
k S_{s . v i r} \lambda(1,1)((X, \Theta))=-k S_{s . g r a v} \lambda(1,1)((X \lambda-1, \Theta \lambda-1))
$$

This last relation is reminiscent of

$$
S_{1}(h \lambda-1)=S_{2}(h),
$$

and explains why one obtains the classical supergravity action from the geometric action when

$$
(X, \Theta) \bullet(\phi, \psi)=(z, \theta)
$$

Finally we obtain the "composition formula",

$$
\begin{aligned}
k S_{\text {s.vir }} \lambda(1,1)((X, \Theta) \bullet(\phi \lambda-1, \psi \lambda-1)) & =-k S_{\text {s.grav }} \lambda(1,1)((\phi, \psi))+k S_{\text {s.vir }} \lambda(1,1)((X, \Theta)) \\
& -k \int d \lambda 2 z d \lambda 2 \theta \frac{D_{\bar{\theta}} \phi-\psi D_{\bar{\theta}} \psi}{\left(D_{\theta} \psi\right) \lambda 2} \mathcal{S}(\Theta) .
\end{aligned}
$$

\section{Induced Quantum $(1,1)$ Supergravity}


In this section we study the quantum theory of $(1,1)$ supergravity using the analysis derived in the previous sections. Initially, it has been demonstrated that the classical geometric action describing the superVirasoro algebra is equivalent to the constrained $(0,1) O S p(1,2)$ WZNW model. However, it has been argued by Ooguri and Bershadsky [19] that the Borel gauged bosonic $O S p(1,2)$ WZNW model at level $k$ gives a theory whose left moving part gives representations of the superVirasoro algebra with central charge,

$$
c_{k}=\frac{3 d_{k}}{2}=\frac{15}{2}-3(2 k+3)-\frac{3}{(2 k+3)},
$$

while the right moving part is described by an $O S p(1,2)$ current algebra. It can easily be deduced that the Borel gauged $(0,1) O S p(1,2)$ WZNW at level $k$ has an $N=1$ $O S p(1,2)$ current algebra in the the right moving sector while the left moving sector is described by a superconformal field theory with central charge given by Eq.(5.1)

Therefore, it can be concluded that quantum mechaniclly, the theory of the superVirasoro group with the geometric action $-\left(d_{k} / 4\right) S \lambda(1,1)_{\text {s.vir }}(\Theta)$ is equivalent to the constrained $(0,1) \operatorname{OSp}(1,2)$ WZNW model of level $k$. The modification of the central charge which multiplies $S \lambda(1,1)_{\text {s.vir }}(\Theta)$ (which is $k$ at the classical level) could also be understood in terms of the Jacobian factor resulting from the change to a superdiffeomorphic invariant measure in the functional integral of $S \lambda(1,1)_{\text {s.vir }}(\Theta)$.

The superstress energy tensor generating the superVirasoro algebra satisfies the operator product expansion

$$
T\left(Z_{1}\right) T\left(Z_{2}\right)=\frac{d_{k} / 4}{Z_{12} \lambda 3}+3 / 2 \frac{\theta_{12} T\left(Z_{2}\right)}{Z_{12} \lambda 2}+\frac{\theta_{12} \partial_{z_{2}} T\left(Z_{2}\right)}{Z_{12}}+1 / 2 \frac{D_{\theta_{2}} T\left(Z_{2}\right)}{Z_{12}},
$$

where $Z$ denotes a point in $(1,1)$ superspace, $Z_{12}=z_{1}-z_{2}-\theta_{1} \theta_{2}$ and $\theta_{12}=\theta_{1}-\theta_{2}$.

In the super light-cone gauge, the effective $(1,1)$ supergravity action is the generating functional for the superstress energy tensor of the supermatter inducing it. The operator product expansion of the superstress tensor implies the following relation for its generating functional $\Gamma\left(H \lambda z_{\bar{\theta}}\right)$,

$$
\left(D_{\bar{\theta}}-H \lambda z_{\bar{\theta}} \partial_{z}+\frac{1}{2}\left(D_{\theta} H \lambda z_{\bar{\theta}}\right) D_{\theta}-\frac{3}{2}\left(\partial_{z} H \lambda z_{\bar{\theta}}\right)\right) \frac{\delta}{\delta H \lambda z_{\bar{\theta}}} \Gamma\left(H \lambda z_{\bar{\theta}}\right)=-\frac{d_{k}}{8} \partial_{z} \lambda 2 D_{\theta} H \lambda z_{\bar{\theta}} .
$$

This is solved by,

$$
\Gamma\left(H \lambda z_{\bar{\theta}}\right)=\frac{d_{k}}{4} S \lambda(1,1)_{s . v i r}(\Theta)
$$

where,

$$
H \lambda z_{\bar{\theta}}=\frac{D_{\bar{\theta}} \phi-\psi D_{\bar{\theta}} \psi}{\left(D_{\theta} \psi \lambda 2\right)}, \quad \Theta(\phi, \bar{z}, \psi, \bar{\theta})=\theta
$$

Therefore, the quantum $(1,1)$ supergravity is defined by the following functional integral,

$$
\int\left[d H \lambda z_{\bar{\theta}}\right] \exp \left(\frac{i d_{k}}{4} S_{s . v i r} \lambda(1,1)(\Theta)\right) .
$$

We will make a change of variable in the above functional integral as follows,

$$
H \lambda z_{\bar{\theta}}+\delta H \lambda z_{\bar{\theta}}=\frac{D_{\bar{\theta}} \phi(z+\delta z, \bar{z}, \theta+\delta \theta, \bar{\theta})-\psi(z+\delta z, \bar{z}, \theta+\delta \theta, \bar{\theta}) D_{\bar{\theta}} \psi(z+\delta z, \bar{z}, \theta+\delta \theta, \bar{\theta})}{\left(D_{\theta} \psi(z+\delta z, \bar{z}, \theta+\delta \theta, \bar{\theta})\right) \lambda 2}
$$


By using the relations

$$
\begin{aligned}
& \phi(z+\delta z, \bar{z}, \theta+\delta \theta, \bar{\theta})=\phi(z, \bar{z}, \theta, \bar{\theta})+\epsilon \lambda z \partial_{z} \phi+\frac{1}{2} D_{\theta} \epsilon \lambda z D_{\theta} \phi \\
& \psi(z+\delta z, \bar{z}, \theta+\delta \theta, \bar{\theta})=\psi(z, \bar{z}, \theta, \bar{\theta})+\epsilon \lambda z \partial_{z} \psi+\frac{1}{2} D_{\theta} \epsilon \lambda z D_{\theta} \psi
\end{aligned}
$$

where

$$
\epsilon \lambda z=\delta z+\theta \delta \theta
$$

is an infinitesimal superdiffeomorphism parameter, we can then write

$$
H \lambda z_{\bar{\theta}}+\delta H \lambda z_{\bar{\theta}}=H \lambda z_{\bar{\theta}}+\left(D_{\bar{\theta}}-H \lambda z_{\bar{\theta}} \partial_{z}+\frac{1}{2}\left(D_{\theta} H \lambda z_{\bar{\theta}}\right) D_{\theta}-\partial_{z} H \lambda z_{\bar{\theta}}\right) \epsilon \lambda z
$$

Therefore, we now have,

$$
\begin{aligned}
& {\left[d H \lambda z_{\bar{\theta}}\right]=\operatorname{sdet}\left(D_{\bar{\theta}}-H \lambda z_{\bar{\theta}} \partial_{z}+\frac{1}{2}\left(D_{\theta} H \lambda z_{\bar{\theta}}\right) D_{\theta}-\partial_{z} H \lambda z_{\bar{\theta}}\right)[d \epsilon \lambda z]} \\
& {\left[d H \lambda z_{\bar{\theta}}\right]=\exp \left(-i \frac{10}{4} S_{\text {s.vir }} \lambda(1,1)(\Theta)\right)[d \epsilon \lambda z]}
\end{aligned}
$$

Thus, the functional integral of $(1,1)$ supergravity can be written as

$$
\int[d \epsilon \lambda z] \exp \left(-i \frac{10-d_{k}}{4} S_{s . v i r} \lambda(1,1)(\Theta)\right)
$$

By noticing that $10-d_{k}=d_{-(k+3)}$, we deduce that induced quantum supergravity theory is equivalent to a Borel gauged $(0,1) O S p(1,2)$ WZNW model at level $k^{\prime}=$ $-(k+3)$. The relation between the level of the current algebra, $k^{\prime}$ and $d_{k}$ agrees with the result calculated using the residual invariance of the theory (Eq. 2.10).

In conclusion, we have demonstrated the equivalence of $S \lambda(1,1)_{\text {s.vir }}$, the geometric action describing superconformal field theory to the theory obtained by gauging the Borel subgroup of the $(0,1)$ supersymmetric $O S p(1,2)$ WZNW model. The action $S \lambda(1,1)_{\text {s.vir }}$ is also understood as a partially gauged $(1,1)$ supersymmetric $O S p(1,2)$ WZNW model in which the residual gauge group transformation becomes the superconformal transformation of the superstress energy tensor of $S \lambda(1,1)_{\text {s.vir }}$. The classical action of $(1,1)$ supergravity in the super light-cone gauge is then derived as a Legendre transform of $S \lambda(1,1)_{\text {s.vir }}$ and the relation between the two actions is a consequence of the properties of WZNW models.

The relation of $(1,1)$ supergravity to that of WZNW model is then exploited to derive the renormalization of the central charge of the current algebra of $(1,1)$ supergravity.

The WZNW models with symmetry group $\mathcal{G}$ can be constructed as two dimensional field theories defined in terms of the standard Kirillov-Kostant symplectic two form [22] on the coadjoint orbit of the $\mathcal{G}$ Kac-Moody group. Therefore, the relation between $S \lambda(1,1)_{\text {s.vir }}$ and the super $O S p(1,2)$ WZNW model reflects the fact that the symplectic structure of the coadjoint orbit of the superVirasoro group is related to that of the $O S p(1,2)$ coadjoint orbit via Hamiltonian reduction $[2,4]$. 
The above analysis can be extended to the more interesting case of $N=2$ supergravity, in which case the supergravity is related to the $N=2$ supersymmetric $O S p(2,2)$ WZNW model. The currents of the $N=2$ super Kac-Moody algebra satisfy non-linear chirality constraints [17] which could be related to the non-linearity of the covariant derivatives in the super light-cone gauge formulation of $(2,2)$ supergravity [18]. We shall report on that in a seperate publication.

\section{ACKNOWLEDGEMENT}

I would like to thank M.B. Green, C. Hull, T. Kuramoto, B. Spence for encouragement and useful conversations.

\section{REFERENCES}

1. A.M. Polyakov, Mod. Phys. Lett. A2 (1987) 893;

V.G. Knizhnik, A.M. Polyakov and A.B. Zamolodchikov, Mod. Phys. Lett. A3 (1988) 819;

A.M. Polyakov and A.B. Zamolodchikov, Mod. Phys. Lett. A3 (1988)1213;

Lectures given by Polyakov at Les Houches summer school on Fields, Strings and Critical Phenomena (1988).

2. A. Alekseev and Shatashvilli, Nucl. Phys B323 (1989) 719.

3. E. Witten, Comm. Math. Phys. 114 (1988) 1.

4. M. Bershadsky and H. Ooguri Comm. Math. Phys. 2(1989) 49.

5. S. Aoyama, Phys. Lett. 228B (1989) 335, S. Aoyama and J. Julve, preprint DFPD/89/TH/60.

6. H. Aratyn, E. Nassimov, S. Pacheva and S. Solomon, Phys. Lett. 234B (1990) 307.

7. M.T. Grisaru and R.M. Xu, Phys. Lett. 205B (1988) 486.

8. T. Kuramoto, Nucl. Phys B346 (1990) 527.

9. S. Aoyama, preprint Padova DEPD/89/TH/75.

10. W.A. Sabra, Mod. Phys. Lett. A6 (1991) 875.

11. G. Delius, P. van Nieuwenhuizen and V.G.J. Rodgers, Int. J. Mod. Phys. A5 (1990) 3943.

12. J. Grundberg and R. Nakayama, Mod. Phys. Lett. A4 (1989) 55.

13. A. M. Polyakov, Int. J. Mod. Phys. A5 (1990) 833.

14. V.G. Kac and T. Todorov, Comm. Math. Phys. 102 (1985) 337.

15. A.M. Polyakov and P.B. Wiegmann, Phys. Lett. 141B (1984) 233.

16. E. Witten, Comm. Math. Phys. 92 (1984) 455.

17. C.M. Hull and B. Spence, Phys. Lett. B241 (1990).

18. A. Alnowaiser, Class. Quantum Grav. 7 (1990) 1033.

19. M. Bershadsky and H. Ooguri, Mod. Phys. Lett, 229B (1989) 374.

20. S.J. Gates, M.T. Grisaru, L. Mezincescu and P.K. Towensend, Nucl. Phys. B286 (1987) 1.

21. V.G. Kac and T. Todorov, Comm. Math. Phys. 102 (1985) 337; Y. Kazama and H. Suzuki, Nucl. Phys. B321 (1989) 232. 
22. A.A. Kirillov, Elements of the Theory of Representations, Springer Verlag (1976).

23. R. Brooks, F. Muhammad and S.J. Gates, Nucl. Phys. B268 (1986) 599.

24. J. Fuchs, Nucl. Phys. B286 (1987) 455.

25. F. Delduc and F. Gieres, preprint CERN-TH 5953/90. 